\title{
BIOMICROSCOPÍA ULTRASÓNICA EN ESCLERECTOMÍA PROFUNDA NO PERFORANTE CON UN NUEVO IMPLANTE ACRÍLICO
}

\section{ULTRASOUND BIOMICROSCOPY IN DEEP SCLERECTOMY WITH A NEW ACRYLIC IMPLANT}

\author{
CONTRERAS $\mathrm{I}^{1,3}$, NOVAL $\mathrm{S}^{1,3}$, MUÑOZ-NEGRETE $\mathrm{FJ}^{2,3}$, REBOLLEDA $\mathrm{G}^{2,3}$, GARCÍA-FEIJOÓ $\mathrm{J}^{2,4}$, \\ DE LA CÁMARA J ${ }^{2,5, *}$
}

\section{RESUMEN}

Objetivo: Describir los hallazgos del examen con biomicroscopía ultrasónica (BMU) de pacientes operados de facoesclerectomía profunda no perforante con un nuevo implante acrílico (Esnoper ${ }^{\circledR}$ ).

Sujetos, materiales y métodos: Exploración con BMU 12 meses después de facoesclerectomía profunda no perforante en tres pacientes con glaucoma crónico de ángulo abierto.

Resultados: Tras un año de seguimiento, los tres pacientes presentan presiones intraoculares menores de $15 \mathrm{mmHg}$ sin tratamiento. En todos ellos, existe un lago intraescleral demostrable por BMU, donde es visible el implante hiperecogénico.

Conclusiones: El nuevo implante no reabsorbible ha sido eficaz a medio plazo en esta serie de casos. La información aportada por BMU ayuda a comprender el mecanismo de acción de la cirugía no perforante.

Palabras clave: biomicroscopía ultrasónica, esclerectomía profunda no perforante, implante acrílico, glaucoma ángulo abierto, facoesclerectomía profunda no perforante.

\begin{abstract}
Objective: To describe the ultrasound biomicroscopic (UBM) characteristics seen in patients who have undergone deep phaco-sclerectomy with a new acrylic implant (Esnoper ${ }^{\circledR}$ ).

Subjects, material and methods: UBM exploration was performed 12 months after deep phacosclerectomy in three patients with chronic open angle glaucoma.

Results: One year after surgery, all three patients had intraocular pressure levels under $15 \mathrm{mmHg}$ and were on no treatment. All of them had an intrascleral space in UBM, in which the hyperechogenic implant was visible.

Conclusions: The new non-absorbable implant has proven to be effective in these cases. Information provided by UBM is useful and assists in understanding the mechanism of action of deep sclerectomy (Arch Soc Esp Oftalmol 2006; 81: 445-450).
\end{abstract}

Key words: ultrasound biomicroscopy, deep sclerectomy, acrylic implant, open angle glaucoma, deep phaco-sclerectomy.

Recibido: 20/1/06. Aceptado: 24/7/06.

Hospital Universitario Ramón y Cajal, Madrid.

1 Licenciado en Medicina.

2 Doctor en Medicina.

3 Hospital Universitario Ramón y Cajal. Madrid.

4 Hospital Universitario San Carlos. Madrid.

5 Hospital Universitario Germans Trias i Pujol. Badalona. Barcelona.

* El Dr. Julio de la Cámara ha diseñado el nuevo implante denominado Esnoper ${ }^{\circledR}$ para la cirugía no perforante. El resto de los autores no tienen ningún interés comercial en ninguno de los productos mencionados en este trabajo.

Correspondencia:

F. J. Muñoz Negrete

Hospital Ramón y Cajal

Carretera Colmenar Viejo, km 9,100, Madrid

España

E-mail: franciscojmunoz@telefonica.net 


\section{INTRODUCCIÓN}

La esclerectomía profunda no perforante (EPNP) es una técnica quirúrgica filtrante diseñada para reducir la presión intraocular (PIO) en pacientes con glaucomas de ángulo abierto no controlados con medicación tópica, que presenta menor número de complicaciones que la trabeculectomía clásica con un adecuado control de la PIO (1-3). El éxito de ambas técnicas continúa siendo comparable cuando se realizan de forma combinada con la cirugía de catarata (4).

El uso de implantes reabsorbibles de ácido hialurónico o de colágeno para conservar el espacio intraescleral, aumenta la tasa de éxito quirúrgico de la EPNP $(5,6)$. Este espacio permanece estable los tres primeros meses, pero seis meses después de la cirugía, es remplazado parcialmente por tejido fibroso hasta en el $27 \%$ de los pacientes, y llega a desaparecer completamente en el 7\% de los casos con implantes de ácido hialurónico, al año del seguimiento (7). Los implantes de colágeno se disuelven entre los 6 y 9 meses (8).

Existen además implantes no reabsorbibles que han demostrado su eficacia a largo plazo en el mantenimiento del efecto hipotensor (9). Recientemente uno de los autores (J.C.) ha desarrollado un nuevo implante no reabsorbible de 2-hidroxietilmetacrilato (Esnoper ${ }^{\circledR}$, AJL Ophthalmics, SA, Miñano, Alava, España) que, al ser un polímero no iónico, muestra una tendencia muy baja a los depósitos de proteínas (fig. 1).

La biomicroscopía ultrasónica (BMU) es una técnica de exploración «in vivo» incruenta que permite visualizar las modificaciones estructurales producidas por las cirugías filtrantes, para comprender sus mecanismos de acción y las causas anatómicas de sus fracasos $(10,11)$.

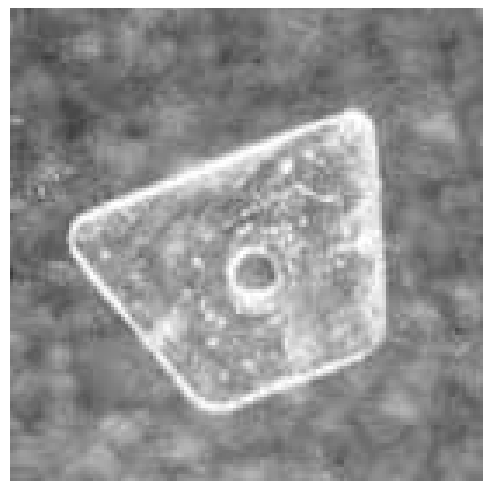

Fig. 1: Implante no reabsorbible de 2-hidroxietilmetacrilato $\left(\right.$ Esnoper $\left.^{\circledR}\right)$.
Presentamos el estudio con BMU de tres pacientes operadas de EPNP con implante de HEMA tras un año de seguimiento.

\section{SUJETOS, MATERIAL Y MÉTODOS}

Tres pacientes con diagnóstico de glaucoma crónico de ángulo abierto con PIO no controlada con medicación tópica y cataratas fueron sometidas a cirugía combinada de facoesclerectomía profunda no perforante (FEPNP) con implante de lente intraocular e implante Esnoper ${ }^{\circledR}$.

La cirugía se realizó bajo anestesia retrobulbar. El procedimiento filtrante se practicó en el cuadrante superior. Tras la realización de un colgajo conjuntival base en fórnix y aplicación de diatermia bipolar suave sobre la superficie escleral expuesta, se disecó un tapete escleral de $5 \times 5 \mathrm{~mm}$ de un tercio del espesor escleral, entrando $1 \mathrm{~mm}$ en córnea clara. A continuación se disecó el colgajo escleral profundo triangular de 4 x $4 \mathrm{~mm}$ y se procedió a realizar la facoemulsificación del cristalino a través de una incisión corneal en meridiano horizontal derecho. La lente intraocular insertada fue acrílica hidrófoba (AcrySof ${ }^{\circledR}$, Alcon, Fort Worth, Texas, USA). Finalizada la cirugía de catarata se procedió a la apertura del canal de Schlemm y disección de la membrana trabeculo-descemética. Posteriormente se realizó la «trabeculectomía ab externo» con pinzas de Mermoud, resección del colgajo profundo y colocación del implante Esnoper ${ }^{\circledR}$ bajo el colgajo superficial, fijándolo a esclera con un punto de nylon 10-0. Por último, se suturó el colgajo escleral superficial con 2 puntos sueltos con nylon 100 y la conjuntiva con 2-4 puntos de nylon 10-0, inyectándose al finalizar el procedimiento una mezcla de corticoide y antibiótico en el espacio subconjuntival.

En el postoperatorio los pacientes fueron tratados con un colirio de antibiótico y corticoide (Polypred $^{\circledR}$, Allergan Inc, Irvine CA, USA) 6 veces al día durante 4-5 semanas. Se realizaron revisiones oftalmológicas los días 1,7 y 30 y a los 3, 6 y 12 meses, recogiendo como parámetros de estudio la agudeza visual (AV), la PIO, el número de fármacos hipotensores, el estado de la ampolla de filtración y la presencia de complicaciones postquirúrgicas.

A los 12 meses, se llevó a cabo la exploración con BMU (UBM 840, Zeiss-Humphrey Medical Instruments, $50 \mathrm{MHz}$ ), con cortes transversales y longitudinales del área quirúrgica. En estas imágenes, se registró la longitud, la anchura y la altura 
máximas del espacio intraescleral, el grosor de la membrana trabéculo-descemética, la presencia y características reflectivas de la ampolla de filtración y la presencia o ausencia de un área hipoecoica supraciliar y/o de un área hiporreflectiva lateral al espacio intraescleral. El implante Esnoper ${ }^{\circledR}$ es hiperreflectivo, lo que permite su visualización.

\section{RESULTADOS}

Las características demográficas y las principales variables prequirúrgicas aparecen en la tabla I. Ninguno de los tres casos presentó complicaciones intra ni postquirúrgicas.

Tras 14 meses de seguimiento la primera paciente (caso 1), presentaba una AV mejor corregida de 0,5 en el ojo operado con una PIO de $15 \mathrm{mmHg} \sin$ tratamiento adicional.

La exploración biomicroscópica demostró la presencia de una ampolla difusa levemente sobreelevada con vascularización similar al resto de la conjuntiva.

En el caso 2, tras un seguimiento de un año, la AV había mejorado a 0,3 , con una PIO de $6 \mathrm{mmHg}$ sin tratamiento y una ampolla de filtración plana, difusa y vascularizada.

La paciente 3,14 meses después de la cirugía, presentaba una AV de 0,2 con una PIO de $14 \mathrm{mmHg}$ sin ampolla apreciable por biomicroscopía.

Las medidas inferidas de las imágenes de BMU al año se presentan en la tabla II. En dos de los casos aparecen área hiporreflectivas laterales al espacio intraescleral y en uno de ellos además un espacio hiporreflectivo supraciliar (fig. 1).

\section{DISCUSIÓN}

Una de las indiscutibles ventajas de la EPNP sobre la trabeculectomía es su mayor seguridad,
Tabla I. Datos prequirúrgicos

\begin{tabular}{lccc}
\hline & Caso 1 & Caso 2 & Caso 3 \\
\hline Edad (años) & 62 & 63 & 83 \\
Sexo & Mujer & Mujer & Mujer \\
Raza & Caucásica & Caucásica & Caucásica \\
Diagnóstico & GPAA & Pseudoexfoliativo & GPAA \\
AV & 0,3 & Cuenta dedos & Cuenta dedos \\
PIO & 22 & 20 & 21 \\
N. ${ }^{\circ}$ fármacos & 2 & 1 & 2 \\
\hline \hline
\end{tabular}

GPAA: Glaucoma primario de ángulo abierto.

relacionada con la baja tasa de complicaciones que presenta (2-4). Además, publicaciones recientes señalan que la EPNP es capaz de conseguir un efecto hipotensor mantenido (1), especialmente si se emplean implantes para prolongar la supervivencia del espacio intraescleral $(5,7,9)$.

Se presenta una serie de tres casos intervenidos de cirugía combinada (FEPNP) con utilización de un nuevo implante de material acrílico no reabsorbible. Con un pequeño volumen de anestesia retrobulbar (no más de $3 \mathrm{cc}$ ), se logra un nivel de analgesia y aquinesia adecuado, lo que hace que la cirugía sea más cómoda para el paciente y más fácil y segura para el cirujano. En todos los casos la PIO al año de seguimiento fue igual o inferior a $15 \mathrm{mmHg}$ sin tratamiento médico adicional. La reducción porcentual promedio de PIO (45\%) es comparable a la publicada con otros implantes $(9,12)$.

La biomicroscopía ultrasónica permite analizar las estructuras del ángulo tras una cirugía filtrante con nitidez cercana a la de estudios histológicos (10). Estudios previos han apuntado que el mecanismo hipotensor de la EPNP puede no ser exclusivamente el drenaje desde el espacio intraescleral a la ampolla de filtración subconjuntival. En la EPNP se crea un espacio intraescleral, separado de la cámara anterior tan sólo por la membrana trabéculo-descemética, detectable por BMU en aproxima-

Tabla II. Características de BMU

\begin{tabular}{lccc}
\hline & Caso 1 & Caso 2 & Caso 3 \\
\hline Anchura & $3,911 \mathrm{~mm}$ & $3,181 \mathrm{~mm}$ & $2,85 \mathrm{~mm}$ \\
Longitud radial & $1,318 \mathrm{~mm}$ & $1,342 \mathrm{~mm}$ & $1,075 \mathrm{~mm}$ \\
Altura & $0,357 \mathrm{~mm}$ & $0,392 \mathrm{~mm}$ & $0,368 \mathrm{~mm}$ \\
Membrana descemética & $0,122 \mathrm{~mm}$ & $0,093 \mathrm{~mm}$ & $0,126 \mathrm{~mm}$ \\
Espacio supraciliar & No & No & Sí \\
Área hiporreflectante lateral & Sín & Sí \\
Ampolla & $15 \mathrm{mmHg}$ & Plana hiporreflectiva & Plana hiporreflectiva \\
PIO postoperatoria (1 año) & $6 \mathrm{mmHg}$ & $14 \mathrm{mmHg}$ \\
\hline \hline
\end{tabular}




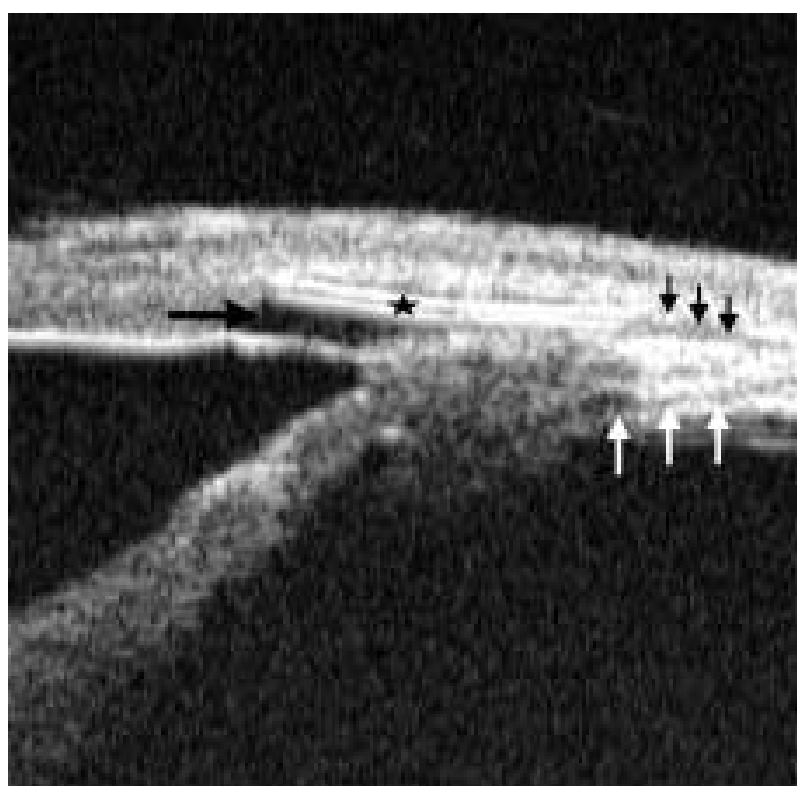

Fig 2: Imagen radial del ángulo en zona quirúrgica del caso 3. * Implante Esnoper ${ }^{\circledR}$. Flecha negra: espacio intraescleral. Flechas negras pequeñas: zona hiporrefléxica intraescleral. Flechas blancas: zona hipoecoíca supraciliar.

damente el $90 \%$ de los casos. A partir de este espacio, el humor acuoso puede filtrarse a través de la fina capa de esclera que cubre el cuerpo ciliar y la coroides al espacio supracoroideo, o bien ser reabsorbido por la formación de nuevos vasos de drena-

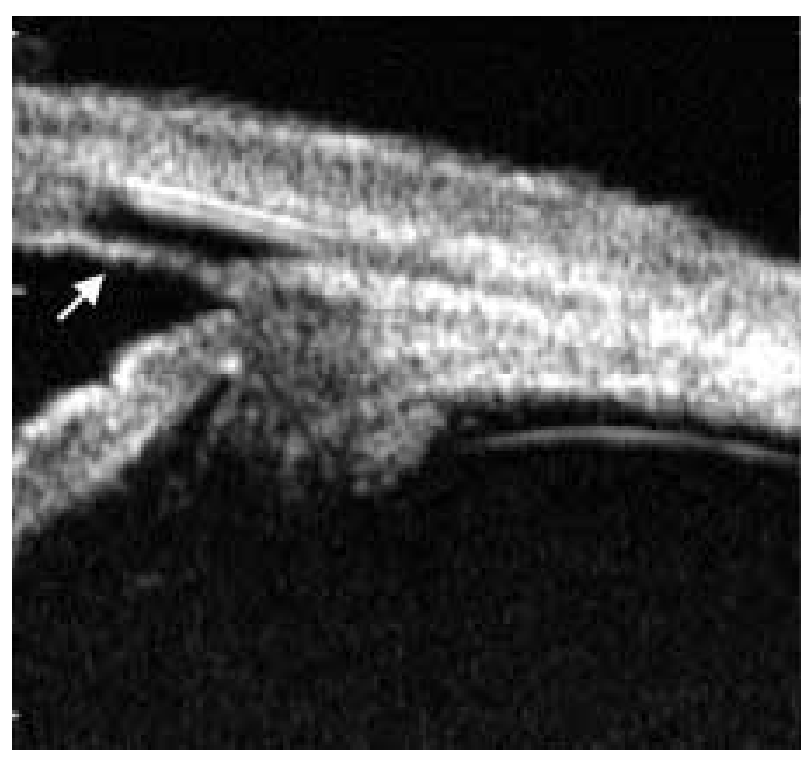

Fig. 3: Imagen radial de la paciente 2 donde observamos que el implante no descansa sobre la membrana trabéculo-descemética (flecha blanca). je o drenar por los orificios del canal de Schlemm y a las venas epiesclerales.

Aunque en prácticamente el $90 \%$ de los casos tras una EPNP se puede detectar una ampolla de filtración, en la mayoría de los casos estas son planas y difusas $(8,13,14)$. Marchini et al (7) encontraron sólo un $60 \%$ de ampollas de filtración tras EPNP con implante de colágeno, sin que su ausencia implicase un mal control tensional. El tipo de ampolla de filtración tampoco parece estar relacionado con el éxito quirúrgico (14). La presencia de un espacio supracoroideo hiporrefléxico en la BMU ha sido interpretado como una señal de filtración a este nivel, siendo detectado entre el 45 y $60 \%$ de pacientes tras EPNP. Pacientes con dicho espacio supracoroideo por BMU presentaban niveles de PIO significativamente menores frente a pacientes sin este signo $(7,8,13)$. También la presencia de tejido escleral hiporreflectivo adyacente al espacio intraescleral, detectable en un $47 \%$ de los casos, parece representar filtración a ese nivel (7). De esta forma, en el trabajo de Marchini et al (7), todos aquellos pacientes con los tres signos de filtración presentes en BMU (ampolla subconjuntival, área hipoecoica supraciliar y tejido hiporreflectivo escleral) fueron clasificados como éxitos quirúrgicos.

En todas nuestras pacientes se pudo localizar por BMU el implante Esnoper ${ }^{\circledR}$, manteniendo el lago intraescleral. En todas se detectó una ampolla de filtración conjuntival; las pacientes 2 y 3 presentaban además un área hiporreflectante adyacente al espacio intraescleral y la paciente 3 un área hipoecoica supraciliar (fig. 2). Las medidas del espacio intraescleral halladas son similares a las de otras series $(7,14)$.

La paciente con mayor reducción de PIO era la que presentaba un menor grosor de la membrana trabéculo-descemética $(0,093 \mathrm{~mm})$.

Ravinet et al (12), encontraron que la tasa de goniopunción en pacientes en los que se había colocado un implante no reabsorbible (T-flux) era mayor que aquellos pacientes sin implante $(63,6 \%$ vs. $36,4 \%)$, aunque la caída de PIO tras ésta era mayor $(6,1 \mathrm{mmHg}$ vs. $3,35 \mathrm{mmHg})$. Especulaban con la posibilidad de que el implante de T-flux produjera una limitación en la filtración a través de la membrana trabéculo-descemética al apoyarse sobre ella. Esta hipótesis se veía reforzada por el hecho de que la goniopunción era menos efectiva cuando se realizaba sobre el implante. 
El nuevo implante también se coloca sobre la membrana trabéculo-descemética, por lo que podría interferir de modo similar con la filtración. Sin embargo, los 3 casos presentaron una PIO inferior o igual $15 \mathrm{mmHg}$ al año de seguimiento sin tratamiento médico y sin precisar goniopunción. De hecho en las imágenes de BMU, el implante se encuentra dentro del lago intraescleral sin apoyarse sobre la membrana trabeculo-descemética (fig. 3).

En resumen, en esta serie inicial el implante de HEMA tiene una buena tolerancia tisular, no observándose efectos adversos. La eficacia hipotensora, gracias a la conservación del espacio intraescleral, se mantiene al menos tras un año de seguimiento. Son precisos estudios más amplios para confirmar la validez del implante. Por otro lado, la BMU resulta una herramienta muy útil en el estudio de las vías de filtración del humor acuoso tras la cirugía filtrante.

\section{BIBLIOGRAFÍA}

1. Shaarawy T, Mansouri K, Schnyder C, Ravinet E, Achache $F$, Mermoud A. Long-term results of deep sclerectomy with collagen implant. J Cataract Refract Surg 2004; 30: 1225-1231.

2. Mermoud A, Schnyder CC, Sickenberg M, Chiou A, Hediguer SE, Faggioni R. Comparison of deep sclerectomy with collagen implant and trabeculectomy in open-angle glaucoma. J Cataract Refract Surg 1999; 25: 323-331.

3. El Sayyad F, Helal M, El-Kholify H, Khalil M, El-Maghraby A. Nonpenetrating deep sclerectomy versus trabeculectomy in bilateral primary open-angle glaucoma. Ophthalmology 2000; 107: 1671-1674.
4. Muñoz Negrete FJ, Rebolleda G, Noval S. Facoesclerectomía profunda no perforante. Resultados y complicaciones. Arch Soc Esp Oftalmol 2003; 78: 499-506.

5. Shaarawy T, Nguyen C, Schnyder C, Mermoud A. Comparative study between deep sclerectomy with and without collagen implant: long term follow up. Br J Ophthalmol 2004; 88: 95-98.

6. Galassi F, Sodi A, Ucci F, Pieri B, Renieri G. Deep sclerectomy in primary open-angle glaucoma: comparison among different implants. Acta Ophthalmol Scand Suppl 2002; 236: 63-64.

7. Marchini G, Marraffa M, Brunelli C, Morbio R, Bonomi L. Ultrasound biomicroscopy and intraocular-pressurelowering mechanisms of deep sclerectomy with reticular hyaluronic acid implant. J Cataract Refract Surg 2001; 27: 507-517.

8. Chiou AG, Mermoud A, Underhahl JP, Schnyder CC. An ultrasound biomicroscopic study of eyes after deep sclerectomy with collagen implant. Ophthlamology 1998; 105: 746-750.

9. Dahan E, Ravinet E, Ben-Simon GJ, Mermoud A. Comparison of the efficacy and longevity of nonpenetrating glaucoma surgery with and without a new, nonabsorbable hydrophilic implant. Opthalmic Surg Lasers Imaging 2003; 34: 457-463.

10. Sánchez Salorio M, García Feijóo J, Pazos González B. Biomicroscopía ultrasónica en oftalmología. Madrid: Tecnimedia Editorial; 1998.

11. Yaramoto T, Sakuma T, Kitazawa Y. An ultrasound biomicroscopic study of filtering blebs after mitomycin C trabeculectomy. Ophthalmology 1995; 102: 1770-1776.

12. Ravinet E, Bovey E, Mermoud A. T-Flux implant versus Healon GV in deep sclerectomy. J Glaucoma 2004; 13:46-50.

13. Kazakova D, Roters S, Schnyder CC, Achache F, JonescuCuypers C, Mermoud A, et al. Ultrasound biomicroscopy images: long-term results alter deep sclerectomy with collagen implant. Graefes Arch Clin Exp Ophthalmol 2002; 240:918-923.

14. Khairy HA, Atta HR, Green FD, van der Hoek J, AzuaraBlanco A. Ultrasound biomicroscopy in deep sclerectomy. Eye 2005; 19:555-560. 